\title{
Investigation of Transverse Oscillation Method
}

\author{
Udesen, Jesper; Jensen, Jørgen Arendt
}

Published in:

I E E E Transactions on Ultrasonics, Ferroelectrics and Frequency Control

Link to article, DOI:

10.1109/TUFFC.2006.1632686

Publication date:

2006

Document Version

Publisher's PDF, also known as Version of record

Link back to DTU Orbit

Citation (APA):

Udesen, J., \& Jensen, J. A. (2006). Investigation of Transverse Oscillation Method. I E E E Transactions on Ultrasonics, Ferroelectrics and Frequency Control, 53(5), 959-971.

https://doi.org/10.1109/TUFFC.2006.1632686

\section{General rights}

Copyright and moral rights for the publications made accessible in the public portal are retained by the authors and/or other copyright owners and it is a condition of accessing publications that users recognise and abide by the legal requirements associated with these rights.

- Users may download and print one copy of any publication from the public portal for the purpose of private study or research.

- You may not further distribute the material or use it for any profit-making activity or commercial gain

- You may freely distribute the URL identifying the publication in the public portal

If you believe that this document breaches copyright please contact us providing details, and we will remove access to the work immediately and investigate your claim. 


\title{
Investigation of Transverse Oscillation Method
}

\author{
Jesper Udesen and Jørgen Arendt Jensen,
}

\begin{abstract}
Conventional ultrasound scanners can display only the axial component of the blood velocity vector, which is a significant limitation when vessels nearly parallel to the skin surface are scanned. The transverse oscillation (TO) method overcomes this limitation by introducing a TO and an axial oscillation in the pulse echo field. The theory behind the creation of the double oscillation pulse echo field is explained as well as the theory behind the estimation of the vector velocity. A parameter study of the method is performed, using the ultrasound simulation program Field II. A virtual linear-array transducer with center frequency $7 \mathrm{MHz}$ and 128 active elements is created, and a virtual blood vessel of radius $6.4 \mathrm{~mm}$ is simulated.

The performance of the TO method is found around an initial point in the parameter space. The parameters varied are: flow angle, transmit focus depth, receive apodization, pulse length, transverse wave length, number of emissions, signal-to-noise ratio (SNR), and type of echo-canceling filter used.
\end{abstract}

Using an experimental scanner, the performance of the TO method is evaluated. An experimental flowrig is used to create laminar parabolic flow in a blood mimicking fluid, and the fluid is scanned under different flow-to-beam angles. The relative standard deviation on the transverse velocity estimate is found to be less than $\mathbf{1 0 \%}$ for all angles between $50^{\circ}$ and $90^{\circ}$. Furthermore, the TO method is evaluated in the flowrig using pulsatile flow, which resembles the flow in the femoral artery. The estimated volume flow as a function of time is compared to the volume flow derived from a conventional axial method at a flow-to-beam angle of $60^{\circ}$. It is found that the method is highly sensitive to the angle between the flow and the beam direction. Also, the choice of echo canceling filter affects the performance significantly.

\section{INTRODUCTION}

$\mathrm{T}$ ODAY ultrasound scanners can display an image of the interior of the human body with a blood velocity image superimposed. However, the scanner does not yield the magnitude of the vector velocity, but only the vector velocity projected onto the axis of the ultrasound beam direction. This causes a severe problem when the angle between the ultrasound beam and the flow is close to $90^{\circ}$ because in this case the blood velocity is not detected. Many authors have addressed this issue, and different methods for calculating the two-dimensional vector velocity have been suggested [1]-[5].

One promising method for calculating the twodimensional vector velocity has been suggested [6]-[8] [transverse oscillation (TO)] and Anderson [9], [10] (spatial quadrature). The method introduces two double oscil-

Manuscript received July 16, 2004; accepted November 12, 2005. The authors are with Technical University of Denmark, DK-2800 Kgs. Lyngby, Denmark (e-mail: ju@oersted.dtu.dk). lating point spread functions (PSFs), each $90^{\circ}$ transverse phase shifted in space, and it uses an autocorrelation estimator to find the velocity. In terms of computation time, the method is superior to existing cross-correlation approaches, e.g., speckle tracking, because only two beamformers in receive are needed.

We previously have shown that the TO method is capable of estimating the velocities in simulations, experiments, and under in-vivo conditions, when the angle between the ultrasound beam and the blood velocity vector is approximately $90^{\circ}[11],[12]$. In order to estimate the vector velocity of the blood, the TO method should be able to give velocity estimates with low bias and standard deviation for any angle. However, a full evaluation of the method has not yet been performed, and a systematic parameter analysis in simulations and experiments still needs to be carried out.

This paper evaluates the TO method. A systematic parameter analysis is performed using the ultrasound simulation program Field II [13], [14] and the the experimental scanner RASMUS [15].

A brief introduction to the method is presented in Section II in which the theory for the field generation is explained, and the autocorrelation velocity estimator is introduced. In Section III the parameter analysis of the TO method is presented. This is done using the Field II program, which is used to simulate signals from a virtual blood vessel. Nine independent parameters then are varied around a fixed point in the parameter space, and the relative standard deviation and relative bias on the estimated transverse velocity $v_{x}$ are calculated. The RASMUS scanner and the flowrig are presented in Section IV along with the other used experimental equipment and the parameters used to perform the experiments. The TO method then is evaluated under different angles of flow in which the flow is laminar, has a parabolic velocity profile, and does not accelerate. The results for this experiment will be discussed in Section V. The TO method will be evaluated in Section VI under more realistic conditions in which the flow resembles the blood velocity in the femoral artery.

\section{THEORY}

In a conventional ultrasound system for blood velocity estimation, the pulse-echo field only oscillates in the axial direction, i.e., along the axis of the ultrasound beam. Blood scatterers passing through the field of interest will produce a signal with a frequency component $f_{z}$ proportional to the axial velocity $v_{z}$, and no knowledge is gained about the transverse velocity component $v_{x}$. This is illustrated 


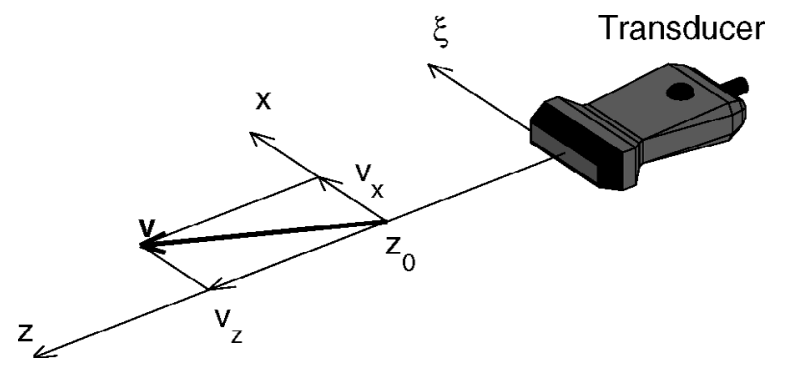

Fig. 1. A conventional ultrasound system will estimate only the axial velocity component $v_{z}$ of a single blood scatterer situated at a depth $z_{0}$ and moving with velocity vector $\mathbf{v}$. The TO method estimates both $v_{x}$ and $v_{z}$. The $\xi$-axis is parallel to the $x$-axis.

in Fig. 1 in which the ultrasound beam propagates along the $z$-axis, and only the velocity component $v_{z}$ along the $z$-axis can be estimated. To overcome this limitation, a new pulse-echo field has to be created.

The basic idea in the TO method is to create a pulseecho field with an oscillation present in both the axial and transverse direction. Blood scatterers traveling through the region of interest will, thus, produce a signal in which two frequency components $f_{x}$ and $f_{z}$ are present. The frequencies $f_{x}$ and $f_{z}$ are related to the velocity components $v_{x}$ and $v_{z}$ by [6], [7]:

$$
\begin{aligned}
& f_{z}=\frac{2 v_{z}}{c} f_{0}, \\
& f_{x}=\frac{v_{x}}{\lambda_{x}},
\end{aligned}
$$

where $c$ is speed of sound, $f_{0}$ is the center frequency of the emitted pulse, and $\lambda_{x}$ is the wave length in the transverse direction of the pulse-echo field. The fundamental acoustic property of grating lobes is used to create the transverse oscillation in the pulse-echo field. This is done by adjusting the apodization of the receive aperture in such a way that the whole aperture resembles two-point sources. Twopoint sources separated in space will give rise to a field in which grating lobes are present, and thereby the grating lobes create the transverse oscillation. Note that this can be done in the receive beamforming, so that the emitted beam in the TO method is similar to the emitted beam in a conventional velocity estimation system.

If the transverse field is assumed to be known, a formal expression for the apodization function can be derived. This is due to the fact that a Fourier relation exists between the apodization function $r(\xi)$ and the transverse field $R(x)$ at the focal point $z_{0}$. Here, $x$ is transverse distance at the focal point and $\xi$ is the distance from the center of the transducer as shown in Fig. 1. The Fourier relation is then $[16]$ :

$$
\begin{aligned}
R(x) & =k_{1} \int_{-\infty}^{\infty} r(\xi) \exp \left(-j \frac{2 \pi}{\lambda_{z} z} x \xi\right) d \xi \\
& =k_{1} \mathcal{F}^{\prime}\{r(\xi)\}
\end{aligned}
$$

where $\mathcal{F}^{\prime}$ is the Fourier transform in which the kernel function uses a spatial frequency variable $1 /\left(\lambda_{z} z\right)$. Here $\lambda_{z}$ is the spatial axial wave length, and $z$ is depth.

In the following, the constant of proportionality $k_{1}$ in (3) is omitted. A transversely oscillating field $R(x)$ with a spatial wave length $\lambda_{x}$ and a lateral width $L$ :

$$
R(x)=\operatorname{rect}(L) \cos \left(2 \pi \frac{x}{\lambda_{x}}\right),
$$

thus yields an apodization function $r(\xi)$ given by:

$$
\begin{aligned}
r(\xi) & =\mathcal{F}^{-1}\{R(x)\} \\
& =\mathcal{F}^{\prime-1}\{\operatorname{rect}(L)\} * \mathcal{F}^{\prime-1}\left\{\cos \left(2 \pi \frac{x}{\lambda_{x}}\right)\right\} \\
& =\frac{L}{2 z \lambda_{z}}\left\{\operatorname{sinc}\left(\pi\left(\frac{\xi}{z \lambda_{z}}+\frac{1}{\lambda_{x}}\right) L\right)\right. \\
& \left.+\operatorname{sinc}\left(\pi\left(\frac{\xi}{z \lambda_{z}}-\frac{1}{\lambda_{x}}\right) L\right)\right\} .
\end{aligned}
$$

Here the operator $*$ denotes spatial convolution. The two sinc functions in (5) have maxima located at:

$$
\frac{\xi}{z \lambda_{z}}= \pm \frac{1}{\lambda_{x}}
$$

so the peak position is at:

$$
\xi_{p}= \pm \frac{z \lambda_{z}}{\lambda_{x}}
$$

The distance $D=\left|2 \xi_{p}\right|$ between the main lobes of the two sinc functions is then related to the transverse wave length $\lambda_{x}$ by the relation

$$
\lambda_{x}=\frac{2 \lambda_{z} z}{D}
$$

The implementation of (5) into the transducers apodization function will yield a pulse-echo field in which an oscillation is present in both the axial and transverse direction. The transverse wave length of the pulse-echo field then can be adjusted by changing the distance between the main lobes of two sinc functions as stated by (8). To ensure that the transverse wave length $\lambda_{x}$ is constant at any depth, the apodization function should be dynamic.

Other apodization functions can be used to create the transverse oscillation. Two delta functions, separated by a distance $D$, will give the most narrow banded transverse field, but the signal-to-noise ratio (SNR) will be poor due to the extensive loss of acoustic energy from using only a small part of the aperture. However, two wide Gaussian functions separated by a distance $D$ would yield a good SNR, but the transverse wave length $\lambda_{x}$ would be poorly defined. Different apodization functions will be investigated further in the next section.

In a conventional flow system, the beamformed in-phase radio frequency (RF) signals are phase shifted $90^{\circ}$ in the axial direction to yield the quadrature signals. Thereby, it 

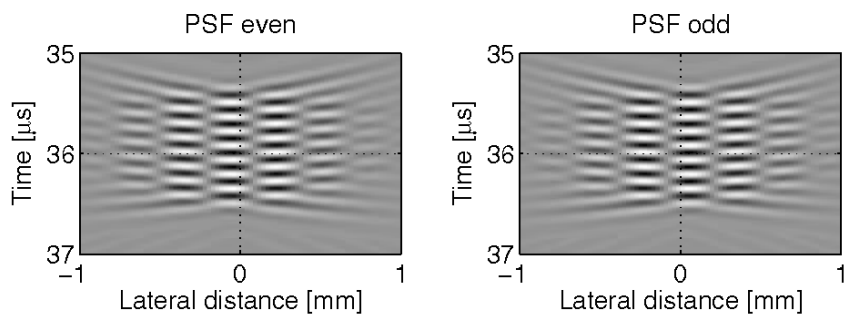

Amplitude spectrum of the PSFs

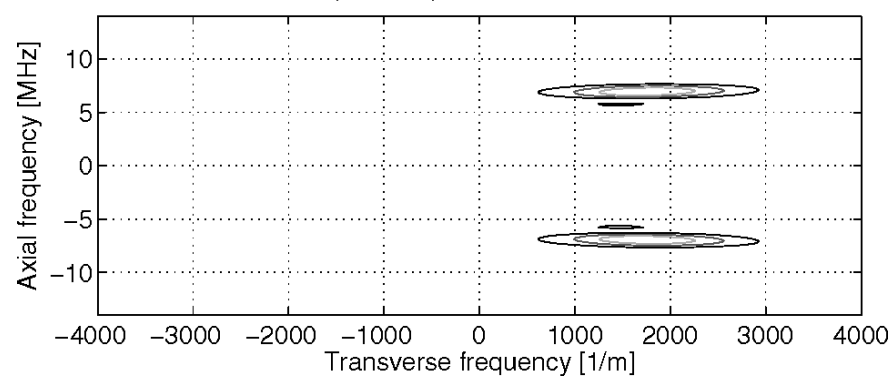

Fig. 2. The two double oscillating PSFs (top) and their corresponding spectrum (bottom) created with the Field II program for a point at $27 \mathrm{~mm}$ using a linear-array transducer with a center frequency of $7 \mathrm{MHz}$. The amplitude spectrum is calculated by taking the twodimensional Fourier transform of $\mathrm{PSF}_{\text {even }}+j \mathrm{PSF}_{\text {odd }}$. Note that the PSFs are $90^{\circ}$ phase shifted with respect to each other and that the amplitude spectrum, therefore, is one-sided.

is possible to determine the sign of the axial velocity that could not be derived from the in-phase signals alone. The TO method makes use of two $90^{\circ}$ phase shifts in spaceone for each spatial direction. This is needed because two frequency components are present in the signals, and hence the phase shift has to be performed in both the axial direction and the transverse direction. The axial phase shift is created using a Hilbert transformation, and the $90^{\circ}$ phase shift in the transverse direction can be accomplished by having two parallel beamformers in receive. The two receive beams are steered so that the transverse distance between each beam is $\lambda_{x} / 4$, which corresponds to a $90^{\circ}$ phase shift in space. This is illustrated in Fig. 2 in which the PSF from each beamformer is shown together with the corresponding spectrum.

When a single blood scatterer travels through the acoustic field with velocity $\mathbf{v}=\left(v_{x}, v_{z}\right)$, the two beamformers in the TO method acquire two signals ${ }^{1}$. Assuming that the signals are sampled at a certain depth with pulse repetition frequency $f_{p r f}$, the signals $r_{\text {even }}(n)$ and $r_{\text {odd }}(n)$ from each beamformer can be modeled ideally as:

$$
\begin{aligned}
r_{\text {even }}(n) & =\cos \left(\theta_{x}(n)\right) \cos \left(\theta_{z}(n)\right), \\
r_{\text {odd }}(n) & =\sin \left(\theta_{x}(n)\right) \cos \left(\theta_{z}(n)\right),
\end{aligned}
$$

\footnotetext{
${ }^{1}$ This discussion of the velocity estimator follows the one given in $[8]$.
}

where the signals for simplicity are assumed to have unit amplitude. The phase factors $\theta_{x}(n)$ and $\theta_{z}(n)$ are given by:

$$
\begin{aligned}
\theta_{z}(n) & =2 \pi f_{z} \frac{n}{f_{p r f}} \\
& =2 \pi \frac{2 v_{z}}{c} f_{0} \frac{n}{f_{p r f}}, \\
\theta_{x}(n) & =2 \pi f_{x} \frac{n}{f_{p r f}} \\
& =2 \pi v_{x} \frac{1}{\lambda_{z}} \frac{n}{f_{p r f}},
\end{aligned}
$$

with $f_{0}$ being the center frequency, $c$ the speed of sound, and the index $n$ the pulse emission number. The factor $\theta_{z}(n)$ is the phase experienced due to a shift in position between the received signals. The phase factor $\theta_{x}(n)$ is the phase of the received signal from a point scatterer sampled with temporal frequency $f_{p r f}$ and traveling with transverse velocity $v_{x}$ through an oscillating field with spatial wavelength $\lambda_{x}$.

Taking the Hilbert transform over the axial distance of (9) and (10) yields:

$$
\begin{gathered}
\hat{r}_{\text {even }}(n)=\cos \left(\theta_{x}(n)\right) e^{j \theta_{z}(n)}, \\
\hat{r}_{\text {odd }}(n)=\sin \left(\theta_{x}(n)\right) e^{j \theta_{z}(n)} .
\end{gathered}
$$

From (15) and (16) two new signals are now formed:

$$
\begin{aligned}
r_{1}(n) & =\hat{r}_{\text {even }}(n)+j \hat{r}_{\text {odd }}(n) \\
& =e^{j\left(\theta_{z}(n)+\theta_{x}(n)\right)}=e^{j \theta_{1}(n)}, \\
r_{2}(n) & =\hat{r}_{\text {even }}(n)-j \hat{r}_{\text {odd }}(n) \\
& =e^{j\left(\theta_{z}(n)-\theta_{x}(n)\right)}=e^{j \theta_{2}(n)} .
\end{aligned}
$$

The phase difference $\Delta \theta_{1}(n)=\theta_{1}(n+1)-\theta_{1}(n)$ and $\Delta \theta_{2}(n)=\theta_{2}(n+1)-\theta_{2}(n)$ between each pulse emission for the signals in (17) and (18) can be estimated using a conventional phase shift estimator [17]. This yields:

$$
\begin{aligned}
\Delta \theta_{z}(n)+\Delta \theta_{x}(n) & =\Delta \theta_{1}(n) \\
& =\arctan \frac{\Im\left\{R_{1}(1)\right\}}{\Re\left\{R_{1}(1)\right\}}, \\
\Delta \theta_{z}(n)-\Delta \theta_{x}(n) & =\Delta \theta_{2}(n) \\
& =\arctan \frac{\Im\left\{R_{2}(1)\right\}}{\Re\left\{R_{2}(1)\right\}},
\end{aligned}
$$

where $R(1)$ is the complex autocorrelation for lag 1 :

$$
\begin{aligned}
& R_{1}(1)=\frac{1}{N-1} \sum_{i=0}^{N-1} r_{1}^{*}(n) r_{1}(n+1), \\
& R_{2}(1)=\frac{1}{N-1} \sum_{i=0}^{N-1} r_{2}^{*}(n) r_{2}(n+1),
\end{aligned}
$$

with $N$ being the number of samples used for each velocity estimate. Rewriting (11) and (14) into the form: 


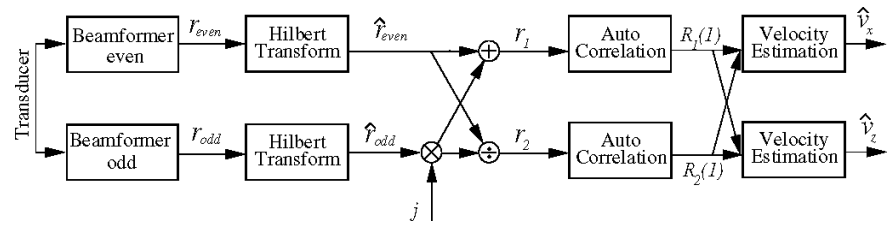

Fig. 3. The block diagram shows data flow from transducer (left) to estimated velocities (right). For simplicity the echo canceling and matched filtration is not shown. The box "Hilbert Transform" represents (16), the box "Auto Correlation" represents (21), and the box "Velocity Estimator" represents (24) and (25).

$$
\begin{aligned}
\Delta \theta_{z}(n) & =\theta_{z}(n+1)-\theta_{z}(n) \\
& =\frac{4 \pi f_{0} v_{z}}{c f_{p r f}}, \\
\Delta \theta_{x}(n) & =\theta_{x}(n+1)-\theta_{x}(n) \\
& =\frac{2 \pi v_{x}}{\lambda_{x} f_{p r f}},
\end{aligned}
$$

and substituting them into (22) and (23), yields two equations with two unknowns. This finally gives ${ }^{2}$

$$
\begin{aligned}
& v_{x}=\frac{\lambda_{x} f_{p r f}}{4 \pi} \times \\
& \operatorname{atan}\left(\frac{\Im\left\{R_{1}(1)\right\} \Re\left\{R_{2}(1)\right\}-\Im\left\{R_{2}(1)\right\} \Re\left\{R_{1}(1)\right\}}{\Re\left\{R_{1}(1)\right\} \Re\left\{R_{2}(1)\right\}+\Im\left\{R_{1}(1)\right\} \Im\left\{R_{2}(1)\right\}}\right), \\
& v_{z}=\frac{c f_{p r f}}{8 \pi f_{0}} \times \\
& \operatorname{atan}\left(\frac{\Im\left\{R_{1}(1)\right\} \Re\left\{R_{2}(1)\right\}+\Im\left\{R_{2}(1)\right\} \Re\left\{R_{1}(1)\right\}}{\Re\left\{R_{1}(1)\right\} \Re\left\{R_{2}(1)\right\}-\Im\left\{R_{1}(1)\right\} \Im\left\{R_{2}(1)\right\}}\right) .
\end{aligned}
$$

The model described by (9) and (10) is valid for a single scatter. However, assuming that the acoustics of the medium is linear, the received signal trace from a collection of scatterers passing through the region of interest will be a sum of the signals from each individual blood scatterer. The model, therefore, also will be valid for a collection of scatterers. A schematic illustration of the velocity estimator is shown in Fig. 3 in which the boxes represent the operations performed by the estimator.

\section{PARAmeter VARIATION}

To optimize the TO method to give the best possible estimate of the velocity vector, a parameter analysis will be presented in this section. The TO method is evaluated by examining the standard deviation and the bias of the estimated velocity. Because the axial velocity can be estimated using a conventional auto correlation algorithm, the focus is on the estimated transverse velocity.

\footnotetext{
${ }^{2}$ Here the relation $\tan (a+b)=(\tan (a)+\tan (b) / 1-\tan (a) \tan (b))$ has been used.
}

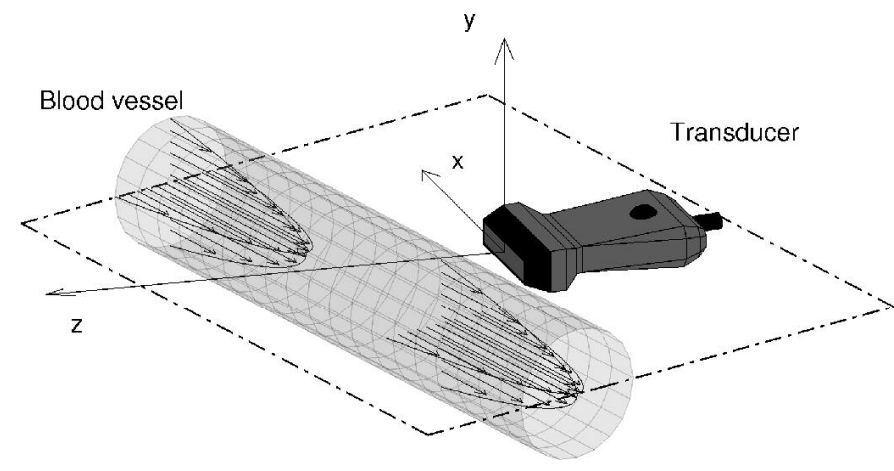

Fig. 4. The setup for the simulations using Field II. The velocity profile in the blood vessel was parabolic, i.e., described by (26).

TABLE I

Transducer Setup.

\begin{tabular}{ll}
\hline Parameter & Value \\
\hline Transducer & Linear array \\
Number of elements & 128 \\
Pitch & $0.208 \mathrm{~mm}$ \\
Kerf & $0.035 \mathrm{~mm}$ \\
Center frequency & $7 \mathrm{MHz}$ \\
Sampling frequency & $100 \mathrm{MHz}$ \\
Apodization in transmit & Hamming \\
\hline
\end{tabular}

The ultrasound simulation program Field II was used to emulate a virtual transducer and blood vessel as seen in Fig. 4. The vessel was placed at a fixed depth of $40 \mathrm{~mm}$ and had a radius of $6.4 \mathrm{~mm}$. It was modeled using 36,255 point scatterers, which were moved with a parabolic velocity profile $v(r)$ given by:

$$
v(r)=v_{0}\left(1-\left(\frac{r}{R}\right)^{2}\right),
$$

here $v_{0}$ is the velocity at the center of the vessel, $r$ is the distance from the center of the vessel to the scatterer moving with velocity $v(r)$, and $R$ is the radius of the vessel. The geometry of the vessel and the peak velocity of the blood are within normal physiological range, e.g., the common carotid artery. However, the parabolic velocity profile is chosen to limit the complexity of the model and cannot be found in the human body. The transducer used in the simulations was a linear-array transducer with 128 active elements in transmit and receive, and the setup parameters can be seen in Table I. The vessel was scanned using 1000 repetitions of the same beam originating from the center of the transducer (the $z$-axis in Fig. 4). After reception of the data, beamforming was performed off-line on a 32 CPU Linux cluster yielding a computation time of approximately 1 week. The different parameters used in the simulations were grouped into two sets: those with fixed values (Tables I and II) and those with values varied during the parameter analysis (Table III). All received 
TABLE II

Parameters That Had Fixed Values in the Simulation.

\begin{tabular}{ll}
\hline Parameter & Value \\
\hline Pulse repetition frequency & $8 \mathrm{kHz}$ \\
Speed of sound & $1540 \mathrm{~m} / \mathrm{s}$ \\
Maximum velocity of blood $v_{0}$ & $1 \mathrm{~m} / \mathrm{s}$ \\
\hline
\end{tabular}

TABLE III

Parameters That Were Varied in the Simulations. ${ }^{1}$

\begin{tabular}{ll}
\hline Parameter & Value \\
\hline Transmit focus & {$[30, \mathbf{4 0}, 50,60] \mathrm{mm}$} \\
Angle of the blood vessel & {$[40,50,60, \mathbf{7 0}, 80,90]^{\circ}$} \\
No. transmit cycles in pulse & {$[4, \mathbf{8}, 12,16]$} \\
Transmit focus & {$[30, \mathbf{4 0}, 50,60] \mathrm{mm}$} \\
Transverse wavelength $\lambda_{x}$ & {$[\mathbf{0 . 8}, 1.1,1.4,1.7] \mathrm{mm}$} \\
Width of each mainlobe & {$[24, \mathbf{3 2}, 48,64]$ Transducer el. } \\
SNR & {$[-30,-24,-18,-12,-6, \infty] \mathrm{dB}$} \\
Pulses per velocity estimate & {$[4,8,16,24, \mathbf{3 2}, 40]$} \\
\hline
\end{tabular}

${ }^{1}$ Values marked with bold face forms the initial point in the parameter space. For the SNR, the initial point is $\infty$.

data were matched filtered before beamforming, and RF averaging was performed [8]. Echo canceling was applied using a simple mean subtraction filter, which removes the zero frequency component in the signal. The filtered signal $y(n)$ is thus:

$$
y(n)=x(n)-\frac{1}{N} \sum_{i=1}^{N} x(i),
$$

where $x(n)$ is the input signal of length $N$.

The TO method is evaluated using the relative standard deviation $\tilde{\sigma}_{v_{x}}$ and relative bias $\tilde{B}_{v_{x}}$ of the estimated transverse velocity. The nondimensional parameters $\tilde{\sigma}_{v_{x}}$ and $\tilde{B}_{v_{x}}$ are defined as:

$$
\begin{aligned}
& \tilde{\sigma}_{v_{x}}=\frac{1}{v_{0}\left(z_{2}-z_{1}\right)} \int_{z_{1}}^{z_{2}} \sqrt{\frac{1}{M} \sum_{i=1}^{M}\left(\hat{v}_{x}^{i}(z)-\bar{v}_{x}(z)\right)^{2}} d z \\
& \tilde{B}_{v_{x}}=\frac{1}{v_{0}\left(z_{2}-z_{1}\right)} \int_{z_{1}}^{z_{2}}\left|\bar{v}_{x}(z)-v_{x}(z)\right| d z
\end{aligned}
$$

where $\hat{v}_{x}^{i}(z)$ is one velocity estimate at a certain depth $z$, $\bar{v}_{x}(z)$ is the mean of the velocity estimates, and $v_{x}(z)$ is the actual velocity described by (26). $z_{1}$ and $z_{2}$, respectively, are the depth to the beginning of the vessel and the end of the vessel, and $M$ is the number of velocity estimates $\hat{v}_{x}^{i}(z)$ used to calculate $\tilde{\sigma}_{v_{x}}$ and $\tilde{B}_{v_{x}}$.

A number of initial conditions were used to form an initial point, which is indicated with bold face in Table III. During the entire parameter analysis, the initial conditions were kept constant except for the parameters, which were
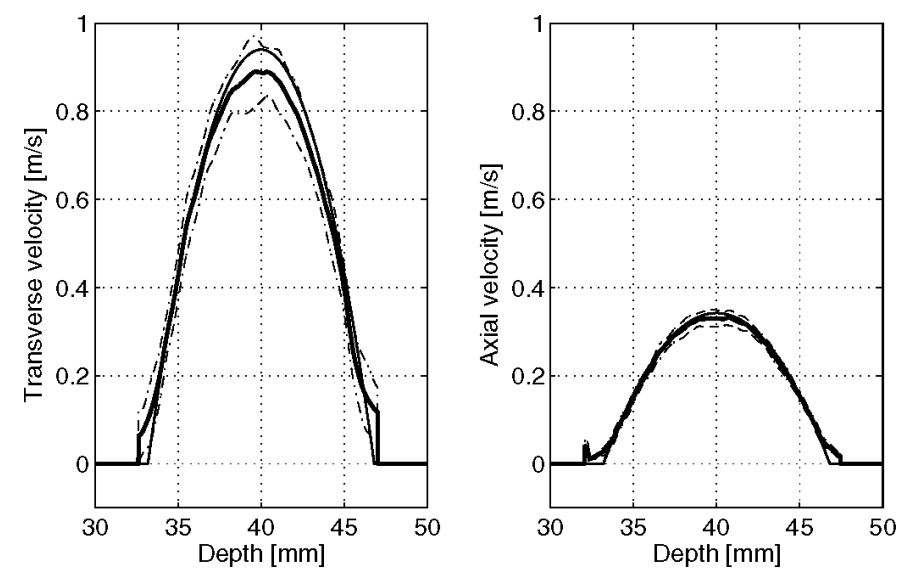

Fig. 5. Left panel: Estimated transverse velocity for the initial point in the parameter space. Right panel: estimated axial velocity for the initial point in the parameter space using a conventional axial estimator. The thick line is the mean of the velocity estimates. The thin line is the actual parabolic profile. The thin, dotted lines are \pm 1 standard deviation.

investigated. Hereby it is ensured that only information concerning the variation of a single parameter at a time was determined.

The receive apodization used at the initial point is not the two sinc functions described by (5) but instead two Hanning functions are used. The Hanning function $h(\xi)$ is defined as:

$$
h(\xi)=\cos ^{2}\left(\frac{\pi \xi}{2 a}\right),-a \leq \xi \leq a,
$$

where $a$ is the width of the Hanning function. The peaks of the two Hanning functions are placed at the positions of the peaks of the sinc functions, and the width of each Hanning function is 32 transducer elements that corresponds to $6.7 \mathrm{~mm}$. In the discussion of the receive parameters, it will be shown that the shape of the two peaks is not crucial for the performance.

For the initial point the relative standard deviation is $\tilde{\sigma}_{v_{x}}=0.061$, the relative bias is $\tilde{B}_{v_{x}}=0.037$, and the corresponding velocity profile can be seen in the left panel in Fig. 5. The estimated transverse velocities for the initial point should be compared with the estimated axial velocity using a conventional axial autocorrelation estimator, and the parameters for the initial point. Here the relative standard deviation is $\tilde{\sigma}_{v_{z}}=0.0134$ and $\tilde{B}_{v_{z}}=0.0068$, and the corresponding velocity plot can be seen in the right panel in Fig. 5. Thus, at a flow angle of $70^{\circ}$, the conventional axial estimator estimates $v_{z}$ approximately six times better than the TO method estimates $v_{x}$.

The transmit parameters are related to the transmit of the ultrasound pulse from the transducer. Three transmit parameters have been investigated:

- Angle between the flow and the ultrasound beam.

- Focus depth of the transmitted ultrasound beam. 

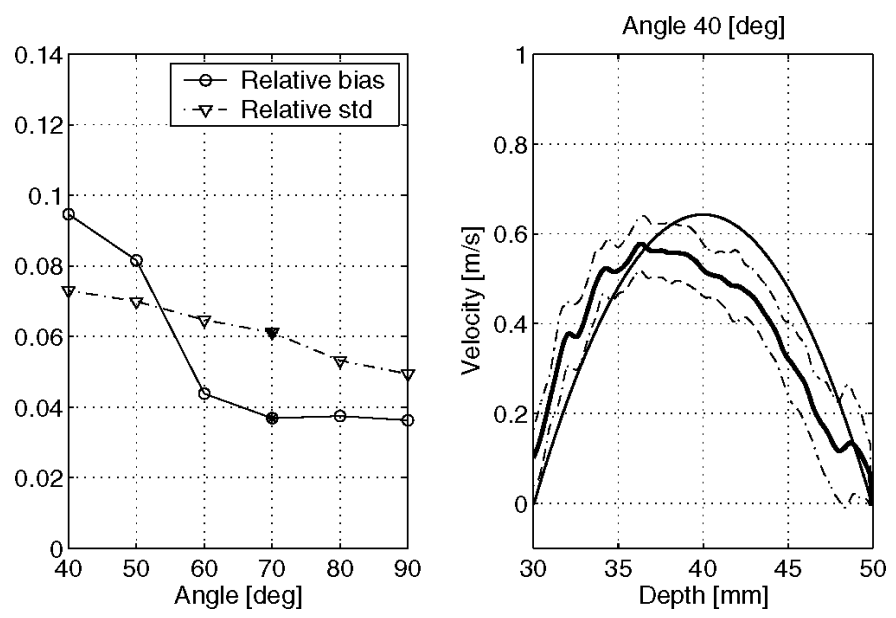

Fig. 6. Left panel: The relative standard deviation and relative bias as a function of flow angle. The initial position is at $70^{\circ}$. Right panel: The estimated transverse velocity and standard deviation at a flow angle of $40^{\circ}$. The thin line indicates the true velocity as a function of depth. The thick line is the estimated mean transverse velocity, and the two dashed lines are the mean transverse velocity \pm 1 standard deviation.

- Number of cycles in the transmitted pulse.

The angle between the ultrasound beam and the flow direction was varied between $90^{\circ}$ and $40^{\circ}$ intervals of $10^{\circ}$, and the corresponding relative standard deviation and relative bias of the transverse velocity were estimated and can be seen in Fig. 6. For purely transverse flow $\left(90^{\circ}\right)$ both the standard deviation and the bias have a minimum, which is expected because only one frequency component is present in the beamformed signals $r_{\text {even }}(n)$ and $r_{\text {odd }}(n)$ at this angle. For angles less than $60^{\circ}$, the bias rises to above 0.08 , and at $40^{\circ}$ the estimated velocity profile differs significantly from the true profile.

The focus depth of the transmitted beam was varied between $30 \mathrm{~mm}$ and $60 \mathrm{~mm}$ in intervals of $10 \mathrm{~mm}$, which can be seen in Fig. 7. Relative standard deviation and relative bias have a minimum when the beam is focused at $60 \mathrm{~mm}$ and $50 \mathrm{~mm}$, respectively, i.e., behind the vessel. This suggests that the TO method performs best when the beam has a relatively broad transverse extend, and hence the transverse spatial frequency $1 / \lambda_{x}$ is well defined and forms a narrow peak in the spectrum. However, focusing the emitted energy in a large area also decreases the returned signal, and hence decreases the SNR, which will increase the bias and the standard deviation. Positioning the focus near the center of the vessel or slightly behind, therefore, will yield the best performance.

The number of cycles in the transmitted pulse has been investigated with the number of cycles ranging from 4 to 16 in intervals of 4 cycles. The corresponding relative standard deviation and relative bias can be seen in Fig. 8. The performance of the TO method is not very sensitive to the number of transmitted cycles. However, the received signal amplitude will scale with the number of transmitted cycles, and hence the SNR in a real scanning situation will increase for a high number of transmitted cycles. The
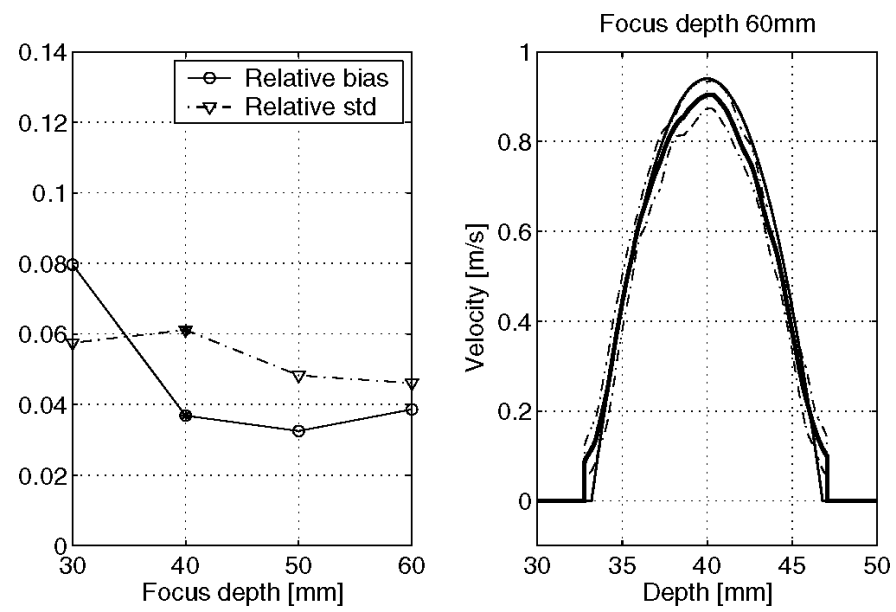

Fig. 7. Left panel: The relative standard deviation and relative bias as a function of transmit focus depth. The initial position is at $40 \mathrm{~mm}$. Right panel: The estimated transverse velocity and standard deviation at a transmit focus depth of $60 \mathrm{~mm}$. The thin line indicates the true velocity as a function of depth. The thick line is the estimated mean transverse velocity, and the two dashed lines are the mean transverse velocity \pm 1 standard deviation.
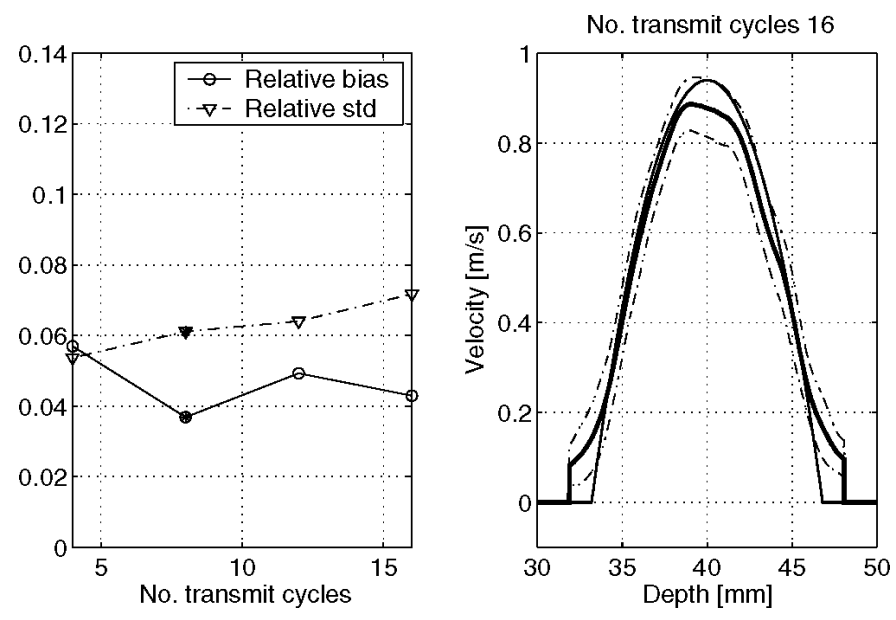

Fig. 8. Left panel: The relative standard deviation and relative bias as a function of number of transmit cycles. The initial position is at 8 cycles. Right panel: The estimated transverse velocity and standard deviation for a 16-cycle, transmitted pulse. The thin line indicates the true velocity as a function of depth. The thick line is the estimated mean transverse velocity, and the two dashed lines are the mean transverse velocity \pm 1 standard deviation.

drawback of having a high number of transmitted cycles is that the axial resolution decreases. A compromise could be to use 12 cycles in the transmitted pulse. This corresponds to an axial extend of $2.6 \mathrm{~mm}$, which is approximately the same size as the transverse extent of the PSF.

The receive parameters characterize the process from the recording of the ultrasound pulse at the transducer to the estimation of the velocity. Six receive parameters have been investigated: 


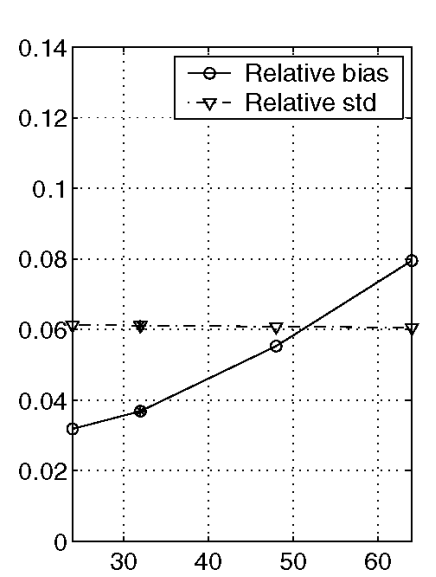

width of each mainlobe in receive apod

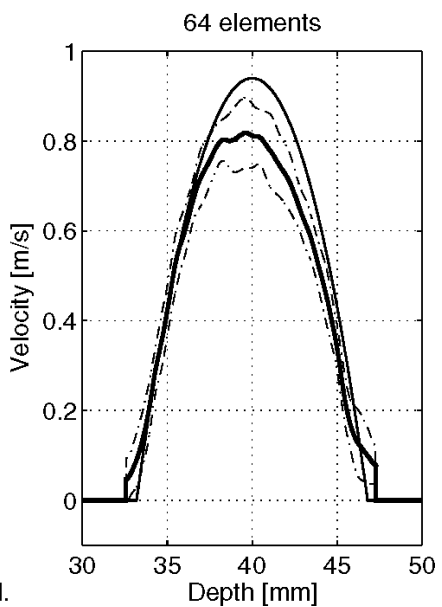

Fig. 9. Left panel: The relative standard deviation and relative bias as a function of the width of each Hanning function used in the receive apodization. The initial position is at 32 elements. Right panel: The estimated transverse velocity and standard deviation when each Hanning is 64 transducer elements in width. The thin line indicates the true velocity as a function of depth. The thick line is the estimated mean transverse velocity, and the two dashed lines are the mean transverse velocity \pm 1 standard deviation.

- Number of transducer elements used to generate each of the two Hanning functions in the receive apodization.

- Transverse wavelength generated in the pulse-echo field.

- Type of echo canceling filter used.

- Number of pulse firings used to make one velocity estimation.

- Type of receive apodization used.

- SNR of received signal.

The width of the two Hanning functions used in receive apodization was varied with the values $(24,32,48,64)$ transducer elements (Fig. 9). Changing the width of each Hanning function seems to have insignificant influence on the relative standard deviation, but it increases the relative bias nearly linearly. This might be due to the fact that a broad Hanning will yield a PSF in which the transverse component is broad banded due to the Fourier relation (3), and hence the velocity estimate will degrade. This effect seems, however, to be present only in the relative bias and not in the relative standard deviation. When the two Hanning functions are broad, the transmitted energy will be large, and hence the SNR in a real scanning situation will increase. A further study of the optimal width of each Hanning, therefore, should be conducted in a noisy environment, e.g., using a real scanner.

The transverse wavelength $\lambda_{x}$ was varied from $0.8 \mathrm{~mm}$ to $1.7 \mathrm{~mm}$ in intervals of $0.3 \mathrm{~mm}$, and the effect of the relative standard deviation and relative bias can be seen in the left panel in Fig. 10. Only the relative standard deviation is significantly effected by the variation in the wavelength, which is a result of the general properties of the autocorrelation estimator. The autocorrelation estimator is unbiased, and the standard deviation is inversely
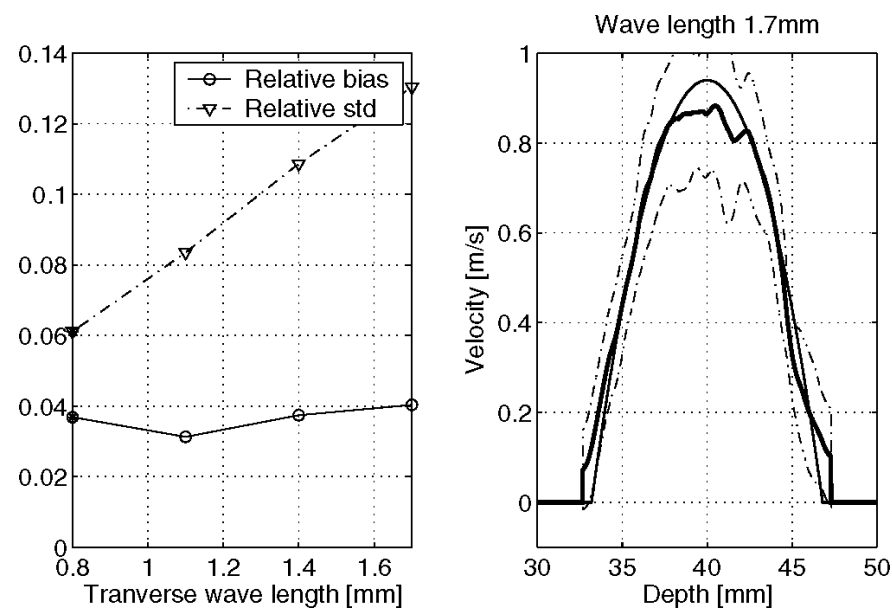

Fig. 10. Left panel: The relative standard deviation and relative bias as a function of the transverse wave length. The initial position is at $0.8 \mathrm{~mm}$. Right panel: The estimated transverse velocity and standard deviation for a transverse wavelength of $1.7 \mathrm{~mm}$. The thin line indicates the true velocity as a function of depth. The thick line is the estimated mean transverse velocity, and the two dashed lines are the mean transverse velocity \pm 1 standard deviation.

proportional to the wavelength [18]. In principle, the relative standard deviation could be decreased below 0.06 if a transverse wavelength shorter that $0.8 \mathrm{~mm}$ was used. However, a short wavelength for a fixed depth $z$ and fixed center frequency $f_{0}$ can be created only by increasing the distance $D$ between the two peaks in the receive apodization function (8). Because the transducer has a fixed width of $26.6 \mathrm{~mm}$, this sets a lower limit to the transverse wavelength.

Different types of echo canceling filters were tested on the received signal and the result can be seen in the left panel in Fig. 11. Stationary tissue with a scattering strength 100 times stronger than the blood signal, has been added in these simulations to make the data more realistic. No echo canceling has been performed in filter $\mathrm{a}$, and filter $\mathrm{b}$ is the mean subtraction filter described by (27). Filter $\mathrm{c}$ has the transfer function $H(z)=1-z^{-1}$, which implies that the filtered signal is simply the result of subtracting consecutive lines. Filter $\mathrm{d}$ has the transfer function $H(z)=\left(1-1.5 z^{-1}+0.5 z^{-3}\right) / 3$. This filter has a more well-defined, cut-off frequency than filter c, which can be seen in Fig. 12.

Because tissue has been modeled in the Field II simulation, the result of having no echo canceling yield a velocity estimate with significant bias. Applying the mean subtraction filter (filter b) described by (27), removes the stationary component, and no distortion on the signals originating from the blood is performed. This filter, therefore, has the lowest relative bias. The relative bias increases for filter $\mathrm{c}$ and filter $\mathrm{d}$, which is a result of the distortion of the frequency spectrum performed by these filters.

The number of pulse firings used for each velocity estimate has been varied between 8 and 40 in intervals of 8 firings. Also, 4 firings for each estimate have been investigated. The result can be seen in the left panel in Fig. 13 . 

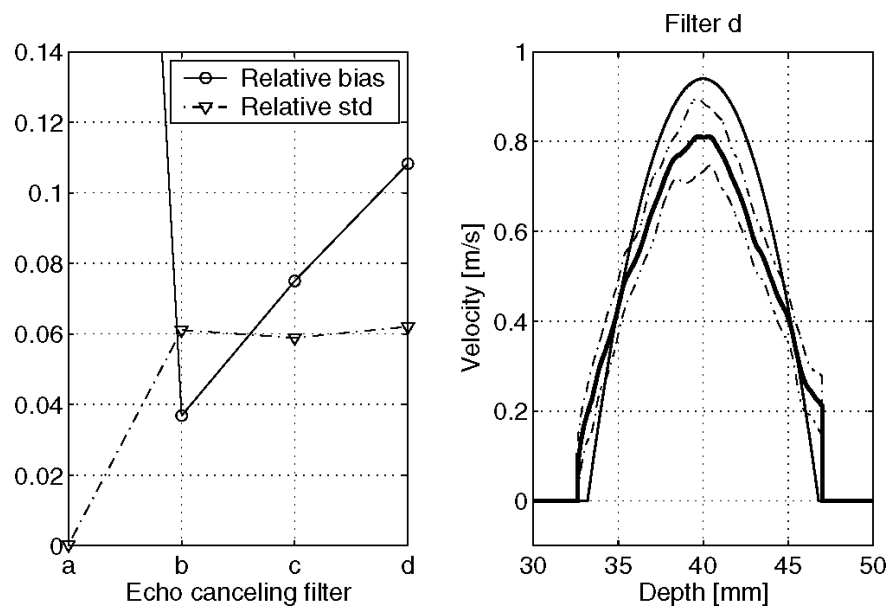

Fig. 11. Left panel: The relative standard deviation and relative bias as a function of different echo-canceling filters. Filter a corresponds to no echo canceling. Here the corresponding bias is 0.63 . Filter $\mathrm{b}$ is the mean subtraction echo canceling described by (27). c is the filter with the transfer function $H(z)=1-z^{-1}$ and d has the transfer function $H(z)=1 / 3\left(1-1.5 z^{-1}+0.5 z^{-3}\right)$. Right panel: The estimated transverse velocity and standard deviation for the filter $\mathrm{d}$ with the transfer function $H(z)=1 / 3\left(1-1.5 z^{-1}+0.5 z^{-3}\right)$. The thin line indicates the true velocity as a function of depth. The thick line is the estimated mean transverse velocity, and the two dashed lines are the mean transverse velocity \pm 1 standard deviation.

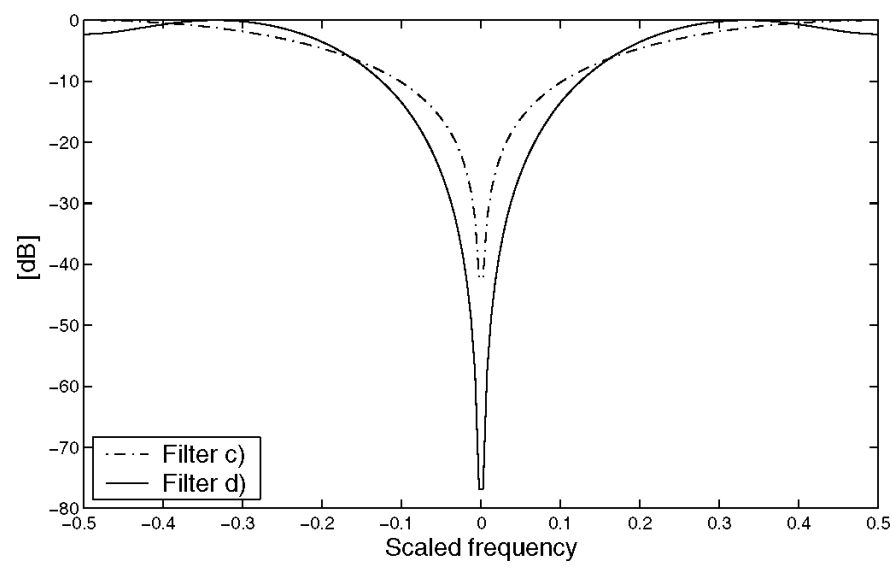

Fig. 12. The frequency characteristics of the echo canceling filters c and $\mathrm{d}$. Filter $\mathrm{c}$ is defined by the transfer function $H(z)=1-z^{-1}$, and filter $\mathrm{d}$ is defined by $H(z)=1 / 3\left(1-1.5 z^{-1}+0.5 z^{-3}\right)$.

The performance of the TO method degrades rapidly as the number of firings per estimate decreases. To obtain a reasonable low bias on the velocity estimate, the number of firings should be at least 8 . This is comparable to the number of firings used in most commercial scanners.

The type of receive apodization has been varied, and the relative standard deviation and relative bias have been measured and are shown in the left panel in Fig. 14. Apodization type a is two Hanning functions $h\left(\xi-\xi_{p}\right)+$ $h\left(\xi+\xi_{p}\right)$ each having the width of 32 transducer elements and center position in $\xi_{p}$. Apodization type b uses two Hamming functions. Apodization type $\mathrm{c}$ is two Blackman functions. Apodization type $\mathrm{d}$ is two Gaussian func-
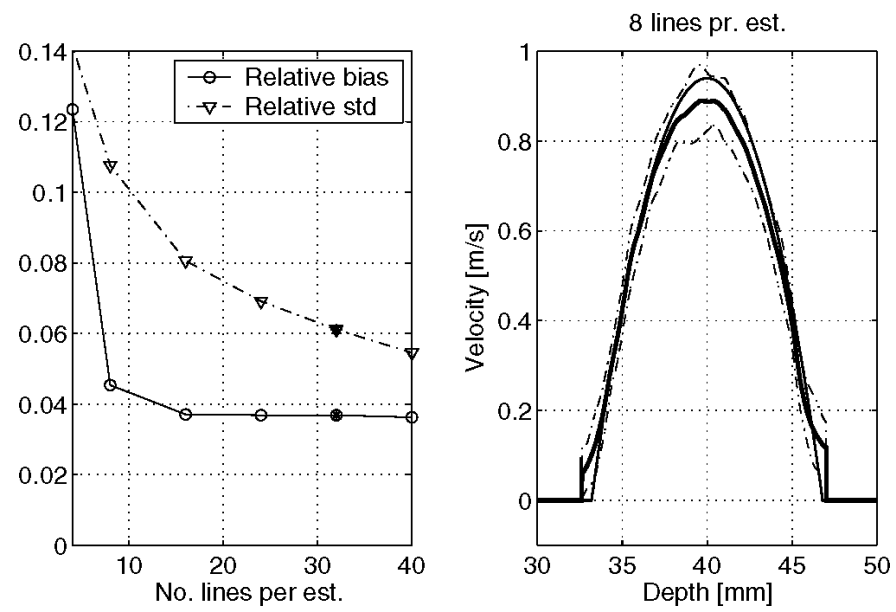

Fig. 13. Left panel: The relative standard deviation and relative bias as a function of number of samples per velocity estimate. The initial position is at 32 lines per estimate. Right panel: The estimated transverse velocity and standard deviation for 8 samples per estimate. The thin line indicates the true velocity as a function of depth. The thick line is the estimated mean transverse velocity, and the two dashed lines are the mean transverse velocity \pm 1 standard deviation.

tions, and apodization type e is two sinc functions as described by (5). Type $\mathrm{f}$ focuses the beam from each of the two receive beamformer along the same line, and uses two different apodization functions on each beamformer. On the even beamformer, two Hanning functions $h\left(\xi-\xi_{p}\right)+h\left(\xi+\xi_{p}\right)$ are used and on the odd beamformer the apodization given by $h\left(\xi-\xi_{p}\right)-h\left(\xi+\xi_{p}\right)$ is used. This is the apodization functions suggested by Anderson [9]. The two apodization functions shift the pulse-echo field $90^{\circ}$ with respect to each other in the transverse direction.

The performance of the TO method does not seem to be effected significantly by the type of apodization. However, because the energy in the received signal will scale with the area under the apodization function, it is advisable to use the functions with the largest area.

The influence of noise on the performance has been tested. Zero mean white Gaussian noise $n(t)$ was added to the received signals $s(t)$ before beamforming, i.e., the noise was added to the signal from each transducer element. The SNR on each element was determined by:

$$
\mathrm{SNR}_{d B}^{e l}=10 \log _{10} \frac{E\left\{s^{2}(t)\right\}}{E\left\{n^{2}(t)\right\}} .
$$

After beamforming and matched filtration, the SNR is [19]:

$\mathrm{SNR}_{d B}^{\text {beam }}=10 \log _{10}\left(\frac{\left|\sum_{k=1}^{N} a(k)\right|^{2}}{\sum_{k=1}^{N}|a(k)|^{2}}\right)+\mathrm{SNR}_{d B}^{\text {mat }}+\mathrm{SNR}_{d B}^{e l}$

where $a(k)$ is the receive apodization on element $k, N$ is the number of transducer elements, which is 128 , and $\mathrm{SNR}_{d B}^{\text {mat }}$ is the improvement in SNR due to matched filtration. Using two Hanning functions as receive apodization, each with a width of 32 transducer elements, yields: 

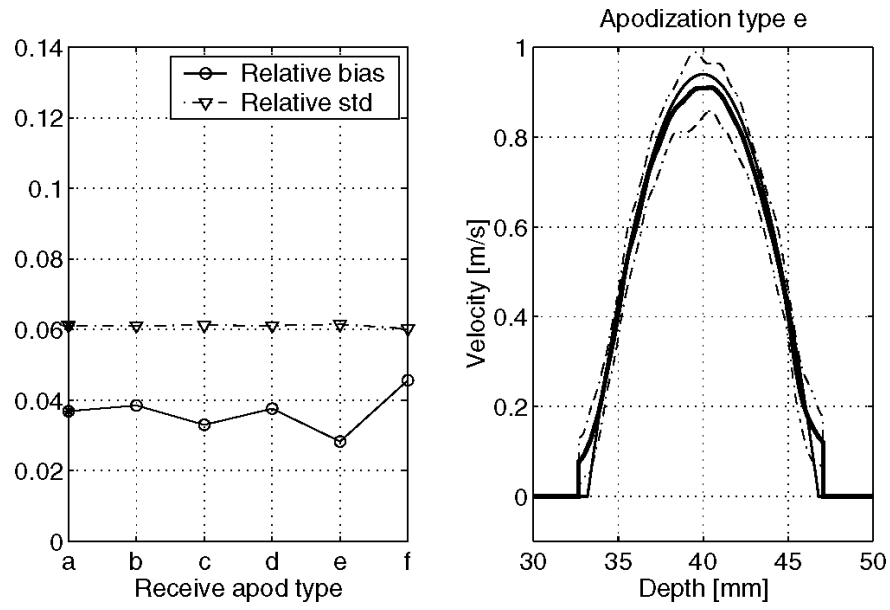

Fig. 14. Left panel: The relative standard deviation and relative bias as a function of the receive apodization type. a is two Hanning functions. $\mathrm{b}$ is two Hamming functions. $\mathrm{c}$ is two Blackman functions. $\mathrm{d}$ is two Gaussian functions, and e is two sinc functions as described by (5). Type $\mathrm{f}$ is the apodization functions suggested by Anderson [9]. The initial position is at apodization type a. Right panel: The estimated transverse velocity and standard deviation for the two sinc apodization in receive. The thin line indicates the true velocity as a function of depth. The thick line is the estimated mean transverse velocity, and the two dashed lines are the mean transverse velocity \pm 1 standard deviation.

$$
\mathrm{SNR}_{d B}^{\text {beam }}=29.2+\mathrm{SNR}_{d B}^{e l},
$$

where the contribution from the beamforming on the SNR is $16.4 \mathrm{~dB}$ and $12.8 \mathrm{~dB}$ from the matched filtration. The influence of $\mathrm{SNR}_{d B}^{e l}$ between $-30 \mathrm{~dB}$ and $0 \mathrm{~dB}$ was investigated in intervals of $6 \mathrm{~dB}$ and is shown in Fig. 15. The performance of the TO method only degrades significantly when the $\mathrm{SNR}_{d B}^{e l}$ is below $-18 \mathrm{~dB}$, which is due to averaging of the noise in the beamforming and the matched filtration. Also, the influence of noise on the velocity estimator presented by Anderson [10] has been investigated. The estimator uses the multiplication of the two signals $\hat{r}_{\text {even }}(n)$ and $\hat{r}_{\text {odd }}(n)$ from (15) and (16) to obtain two signals, which depends only on the axial velocity and the transverse velocity, respectively. It is tempting to split the received signal into a signal that depends on the axial velocity, and a signal that depends on the transverse velocity. Echo canceling can be applied on each signal, and the frequencies originating from the axial movement of the tissue can be removed without destroying the frequencies originating from the transverse movement of the blood. However, multiplying two stochastic signals is not in general advisable, and the relative standard deviation and relative bias rise significantly, when noise is present, which can be seen in Fig. 15.

\section{The Experimental Setup}

The TO method was evaluated on a circulating flowrig, which pumps blood mimicking fluid around a closed circuit. To avoid entrance effects in the flow, the fluid was first led through a $1.2-\mathrm{m}$ long inflow pipe with radius
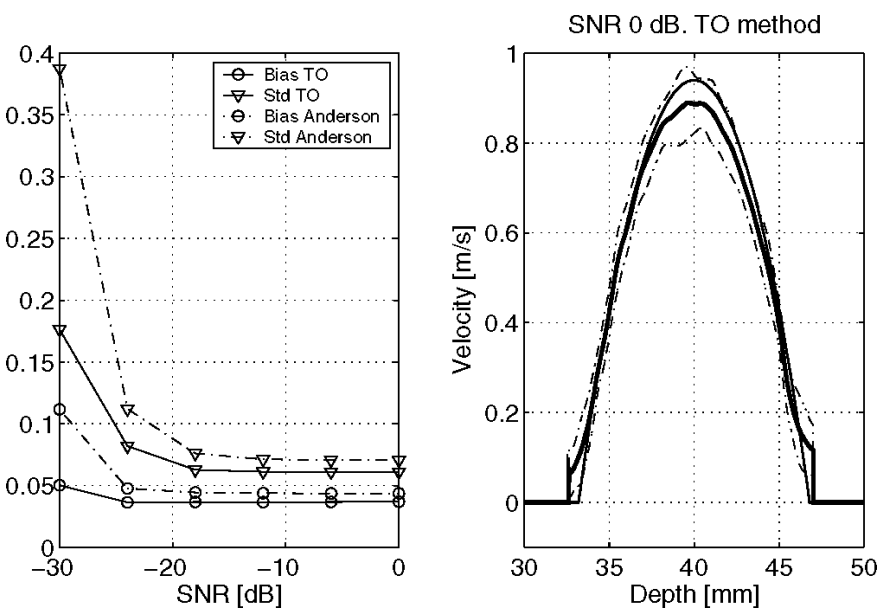

Fig. 15. Left panel: The relative standard deviation and relative bias as a function of SNR for white Gaussian noise added to the channel data before beamforming. The dotted line marked with "o" is the relative standard deviation on the velocity estimates using the TO velocity estimator, and the thick line marked with "o" is the corresponding relative bias. The dotted line marked with " $\nabla$ " is the relative standard deviation on the velocity estimates using the velocity estimator described by Anderson [10], and the thick line marked with " $\nabla$ " is the corresponding relative bias. Right panel: The estimated transverse velocity and standard deviation for the TO estimator with a SNR of $0 \mathrm{~dB}$. The thin line indicates the true velocity as a function of depth. The thick line is the estimated mean transverse velocity, and the two dashed lines are the mean transverse velocity \pm 1 standard deviation.

$R=6.4 \mathrm{~mm}$. At the end of the inflow pipe, the fluid was led through a heat-shrink tube submerged in a water container and scanned. The tube had an internal radius of $6.4 \mathrm{~mm}$ and the walls were $0.5 \mathrm{~mm}$ thick. The fluid volume flow $Q$ was measured using a MAG 1100 flow meter (Danfoss, Hasselager, Denmark), which was situated after the heat shrink tube.

For the experiments, when the fluid velocity was constant over time, a Cole Parmer (Vernon Hills, IL) 7521160 centrifugal pump was used. In this case the bloodmimicking fluid consisted of water, glycerol, orgasol, Triton x-100 (Dansk Fantom Service, Frederikssund, Denmark), NaBenzoat and $\mathrm{K}_{2}$ EDTA diluted 10 to 1 with demineralized water. The blood-mimicking fluid had viscosity $\mu=2.6 \cdot 10^{-3} \mathrm{~kg} /(\mathrm{m} \mathrm{s})$, density $\rho=10^{3} \mathrm{~kg} / \mathrm{m}^{3}$, and the temperature during scanning was $T_{0}=24^{\circ} \mathrm{C}$.

For the experiments in which the flow resembled the human femoral artery, a programmable Compuflow1000 flow pump from Shelley (Toronto, ON, Canada) was used. In this case the blood-mimicking fluid is described in [20], and the temperature during scanning was $T_{0}=24^{\circ} \mathrm{C}$.

To avoid turbulence in the fluid, the Reynolds number should be kept below approximately 2000 [21]. For flow in a pipe, the Reynolds number $R_{e}$ is defined as:

$$
R_{e}=\frac{2 R \rho}{\mu} \bar{v},
$$

where $\bar{v}$ is the mean velocity in the tube. Because $\bar{v}$ was kept below $0.4 \mathrm{~m} / \mathrm{s}$ in all measurements, it was ensured that no turbulence was present in the flow. 
TABLE IV

Transducer Setup.

\begin{tabular}{ll}
\hline Parameter & Value \\
\hline Transducer & Linear array \\
Elevation focus & $25 \mathrm{~mm}$ \\
Number of elements & 128 \\
Pitch & $0.208 \mathrm{~mm}$ \\
Kerf & $0.035 \mathrm{~mm}$ \\
Center frequency & $7 \mathrm{MHz}$ \\
Sampling frequency & $40 \mathrm{MHz}$ \\
Apodization in transmit & Hamming \\
Number of cycles & 8 \\
\hline
\end{tabular}

A $7 \mathrm{MHz}$ linear-array transducer characterized by the parameters in Table IV was used to scan the blood mimicking fluid. The emitted pulse had a center frequency of $7 \mathrm{MHz}$. The pulse was focused at $40 \mathrm{~mm}$ at the center of the vessel, and Hamming apodization was applied on the transmit aperture in order to decrease sidelobe levels. The scanner used was the experimental scanner RASMUS which has 128 transmit channels and 64 receive channels with $2: 1$ multiplexing in receive. The sampling frequency is $40 \mathrm{MHz}$, and the dynamic range is 12 bits for each channel. Channel data were beamformed offline using a $32 \mathrm{CPU}$ cluster system running Matlab (Mathworks, Inc., Natick, MA) under Linux. To acquire data from 128 elements in receive, two consecutive shots were used. Each sampled on the left and right halves of the transducer, respectively; thereafter, the data were combined to form a complete data set of 128 elements. The success of this interleaving procedure relies on the fact that the blood scatters move a distance smaller than the width of the point-spread function during two firings. Consecutive signals from the scatters, therefore, can be added, but the effective $f_{p r f}$ will be decreased by a factor of 2 .

The transverse oscillation in the pulse-echo field was introduced by manipulating the receive apodization function as described in Section II. Two Hanning functions, each with a width of 32 transducer elements, were used to create the receive apodization. The distance between the peaks of the two Hanning functions were adjusted so that the transverse oscillation length $\lambda_{x}$ in the pulse-echo field was kept constant $\lambda_{x}=1 \mathrm{~mm}$ for the entire scanned area. Two beams were then beamformed, each separated a transverse distance of $\lambda_{x} / 4=0.25 \mathrm{~mm}$ using dynamic focusing. The beamforming was performed using a sound speed of $c=1480 \mathrm{~m} / \mathrm{s}$ equal to the speed of sound in water. Echo canceling was applied on all beamformed data using the mean subtraction filter given by (27).

To verify that the TO method can produce a pulse-echo field in which an oscillation is present in the transverse direction, a wire phantom was scanned using the RASMUS scanner and the linear-array transducer described in Table IV. An active aperture consisting of 64 elements was
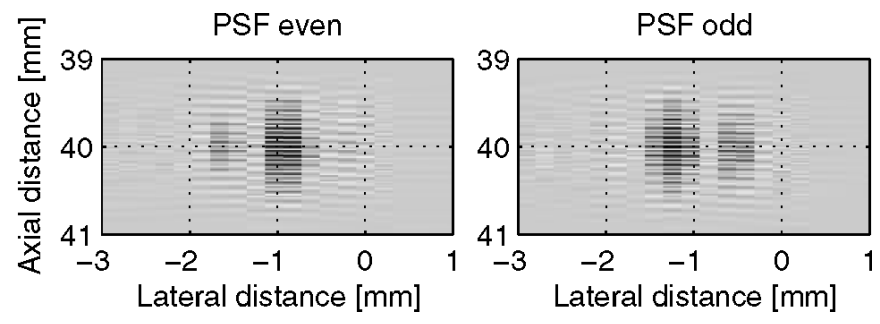

$12 \mathrm{~dB}$ contours.

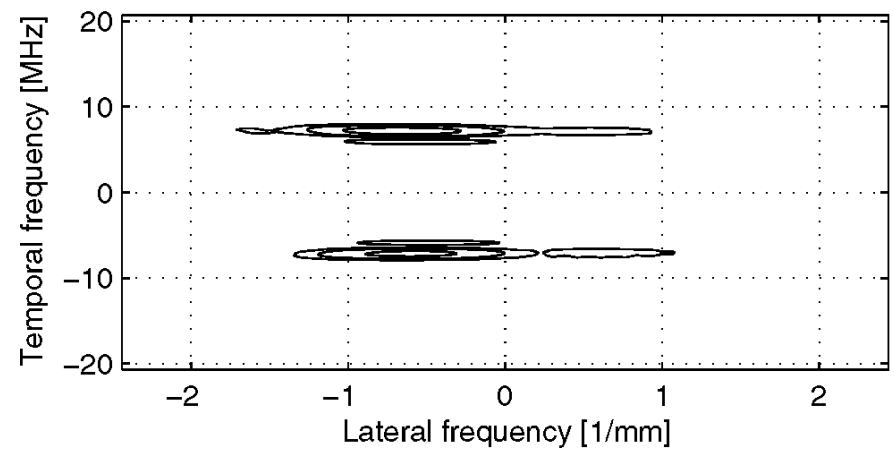

Fig. 16. Top: The PSFs from each beamformer. Bottom: the twodimensional power spectrum of $\mathrm{PSF}_{\text {even }}+j \mathrm{PSF}_{\text {odd }}$.

moved over the transducer surface in steps of one element, and a B-mode image was acquired without envelope detection. The wire was located in water at a depth of $40 \mathrm{~mm}$, and the distance between the maxima of the two Hanning functions in the receive apodization function was adjusted to give a constant transverse wavelength $\lambda_{x}=1.4 \mathrm{~mm}$.

The resulting PSFs for each beamformer can be seen in the top row of Fig. 16. Because the distance between each transducer element was $0.208 \mathrm{~mm}$, the lateral resolution is poor; however, the transverse oscillation is clearly visible.

In the bottom of Fig. 16, the two-dimensional fast Fourier transform (FFT) power spectrum for the two PSFs is shown. The two clearly defined peaks on the $y$-axis is the center frequency of the emitted pulse. The center frequency on the $x$-axis corresponds to the transverse oscillation in the pulse-echo field. From the power spectrum, the mean lateral wavelength is calculated to be $\lambda_{x}=1.6 \mathrm{~mm}$, which is $16 \%$ more than the theoretical value of $\lambda_{x}=1.4 \mathrm{~mm}$. Because the transverse velocity scales with the transverse spatial frequency $1 / \lambda_{x}$, it, therefore, is expected that the velocity estimates will be slightly biased.

\section{Results for Constant Volume Flow}

The first set of measurements was performed on a flow in which no acceleration was present. The volume flow rate $Q$ was fixed at $23.6 \mathrm{ml} / \mathrm{s}$, and since:

$$
Q=\bar{v} A,
$$

where $A=\pi R^{2}$ is the area of the cross section of the pipe, it follows from (34) that the Reynolds number is approximately 900 . The flow, therefore, can be assumed 

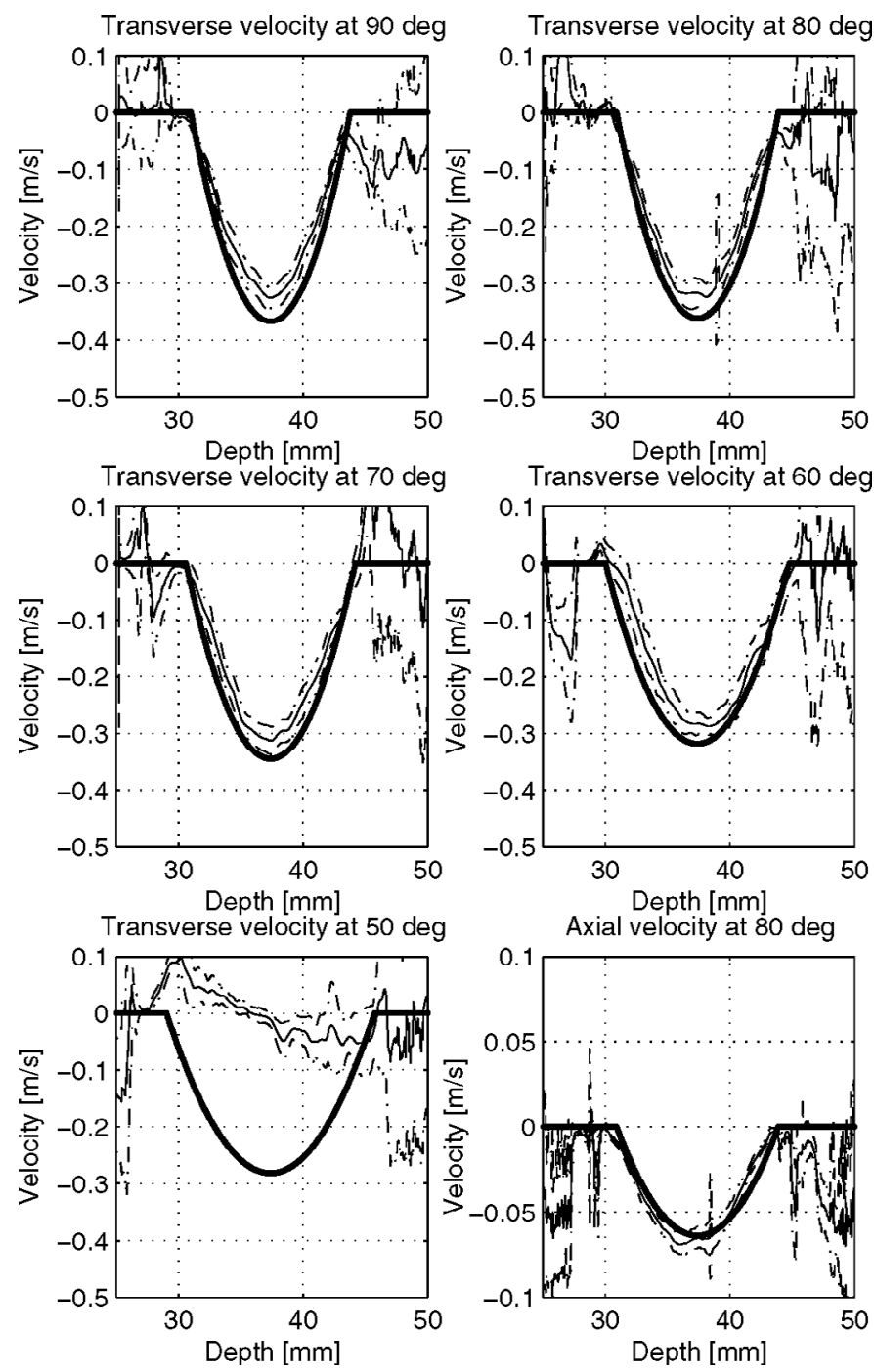

Fig. 17. The velocity as a function of depth for different angles of flow. The thick solid line is the theoretic parabolic velocity profile described by (26). The thin solid line is the mean of all the velocity estimates, and the two dotted lines are the mean profile plus/minus 1 standard deviation. No attempt has been made to suppress false velocity estimates at the vessel wall and in the surrounding water.

laminar, and the flow velocity $v$ as a function of distance $r$ from the center of the pipe can be described by (26). The maximum velocity $v_{0}$ at the center of the pipe was, thus, $v_{0}=0.367 \mathrm{~m} / \mathrm{s}$.

Five experiments were performed in which the angle between the ultrasound beam and the flow velocity vector was varied between $50^{\circ}$ and $90^{\circ}$ in steps of $10^{\circ}$. The $f_{p r f}$ used was $4 \mathrm{kHz}$, and the number of shots fired was 2000 . Due to the interleaving procedure described in the previous section, this gives an effective $f_{p r f}$ of $2 \mathrm{kHz}$ and 1000 beamformed lines, where 32 lines were used for each velocity estimate. In Fig. 17 the transverse velocity profiles and standard deviation as a function of depth are shown for the measured angles of flow. It can be seen that the estimated transverse velocities are approximately parabolic down to a flow angle of $60^{\circ}$. At $50^{\circ}$ no parabolic velocity profile can be seen, even though the standard deviation

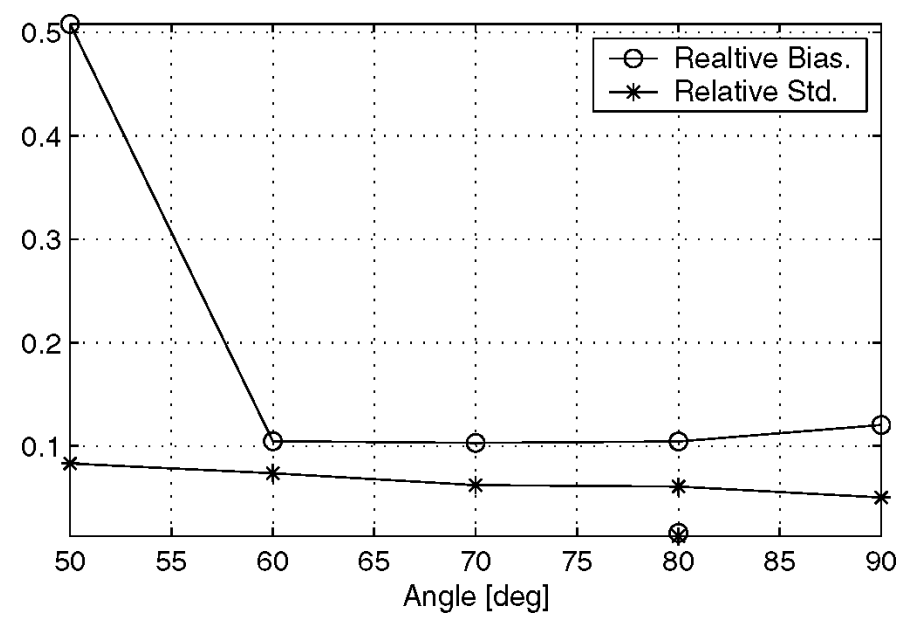

Fig. 18. The relative standard deviation and relative bias on the estimated velocity for different angles of flow.

still remains relatively small. The increase in bias at this angle is in agreement with the simulated results.

For comparison, the estimated axial velocity for $80^{\circ}$ also is shown in Fig. 17 in which the estimate is based on a conventional axial autocorrelation estimator. The angle of $80^{\circ}$ is the smallest of the measured angles in which no aliasing occur in the axial estimate using $f_{p r f}=2 \mathrm{kHz}$. It should be noted that the conventional axial estimate is based on the same channel data as the transverse velocity estimate at the same angle. Only the beamforming is different because no transverse oscillation in the pulse-echo field has been introduced. Hamming apodization was used to suppress sidelobes in the receive beamforming, and 32 beamformed lines were used for each velocity estimate.

To quantitively compare the different velocity measurements, the relative standard deviation $\tilde{\sigma}_{v_{x}}$ and relative bias $\tilde{B}_{v_{x}}$ were estimated. In Fig. $18 \tilde{\sigma}_{v_{x}}$ and $\tilde{B}_{v_{x}}$ are shown, and it can be seen that both $\tilde{B}_{v_{x}}$ and $\tilde{\sigma}_{v_{x}}$ remains approximately constant between $60^{\circ}$ and $90^{\circ}$. The two measurements at $80^{\circ}$, which are not connected by a line, are the relative bias and relative standard deviation for the axial velocity estimate at this angle. Their values are $\tilde{\sigma}_{v_{z}}=0.01$ and $\tilde{B}_{v_{z}}=0.01$, i.e., approximately five times smaller than the corresponding $\tilde{\sigma}_{v_{x}}$ and $\tilde{B}_{v_{x}}$ for the transverse velocity at the same angle. This is in agreement with the corresponding simulations for constant flow.

\section{Results for Pulsatile Flow}

Because the blood flow in the human body is not generally constant, the TO method has been tested for a pulsatile flow, which resembles flow in the femoral artery. The transverse flow velocity was estimated as a function of depth and time for 1.44 seconds of flow; and because the cycle period of the pump was 0.84 seconds, the measurements captured nearly two cycles of flow. At an $f_{p r f}$ of $6 \mathrm{kHz}, 10,080$ beams were emitted from the transducer. Due to the interleaving procedure, this gives an effective 

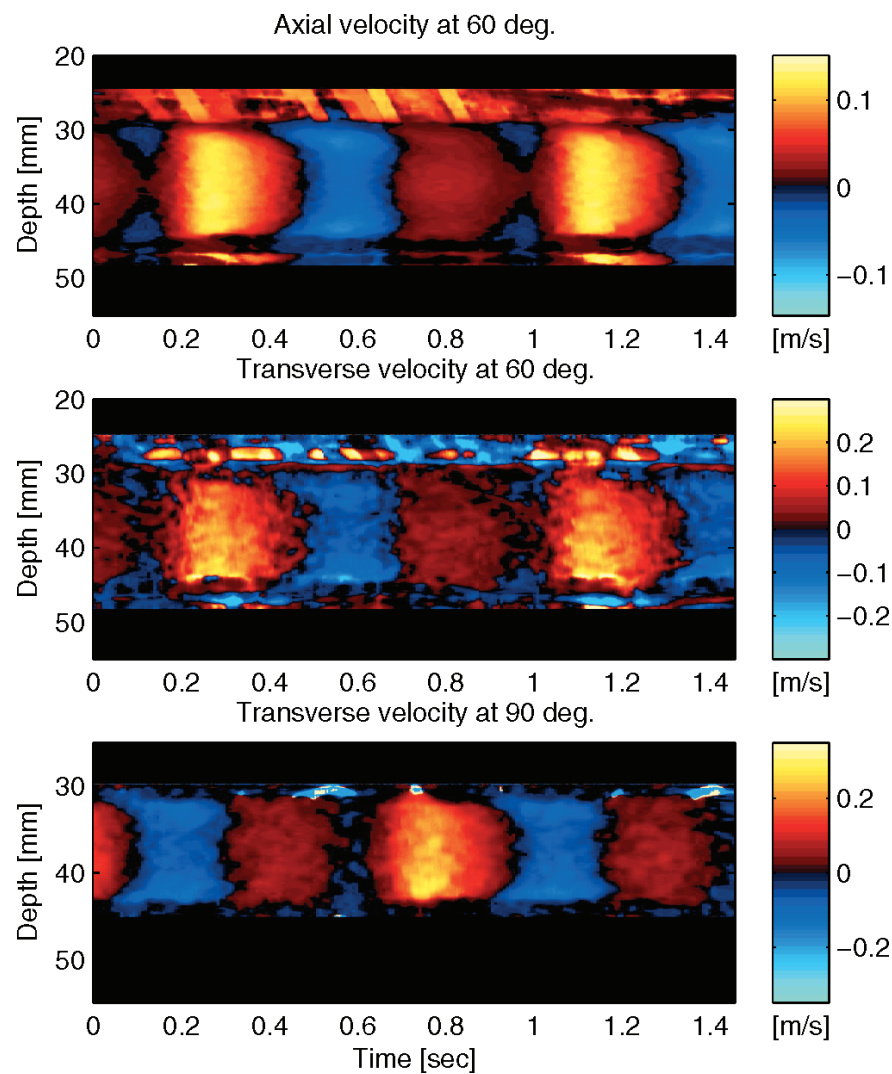

Fig. 19. Velocity as a function of time and depth. Top: Axial velocity at $60^{\circ}$ flow angle. Center: Transverse velocity at $60^{\circ}$ flow angle. Bottom: Transverse velocity at $90^{\circ}$ flow angle. The flow resembles the flow in the human femoral artery. No attempt has been made to suppress false velocity estimates at the vessel wall and in the surrounding water.

$f_{p r f}=3 \mathrm{kHz}$ and 5040 beamformed lines. The lines were divided into segments of 100 lines in which consecutive segments had an overlap of $90 \%$. The segments then were echocanceled using (27) and passed to the transverse velocity estimator.

In Fig. 19 the transverse velocity as a function of depth and time is shown for the angles $60^{\circ}$ and $90^{\circ}$. Also, the axial velocity estimated at $60^{\circ}$ is shown. The axial estimate at $60^{\circ}$ is derived using a conventional axial estimator, and the data used are the same as the transverse velocity estimate at $60^{\circ}$ is based on. Even though the transverse velocity estimates at $90^{\circ}$ or $60^{\circ}$ does not become as good and detailed as the axial estimate at $60^{\circ}$, the transverse estimates still show the same overall flow.

The measurements can be more easily compared by deriving the corresponding volume flow as a function of time, by integrating the velocity over the vessel cross-section area. Here the velocity profile is assumed circular symmetric around the center of the pipe. The volume flow rate is shown as a function of time in Fig. 20. When derived from the axial velocity, the measured volume flow at $60^{\circ}$ clearly shows the characteristic profile of the flow in the femoral artery. Here a large, positive peak in the flow rate is followed by a smaller negative peak and an even smaller positive peak. This pattern also is seen when the flow rate

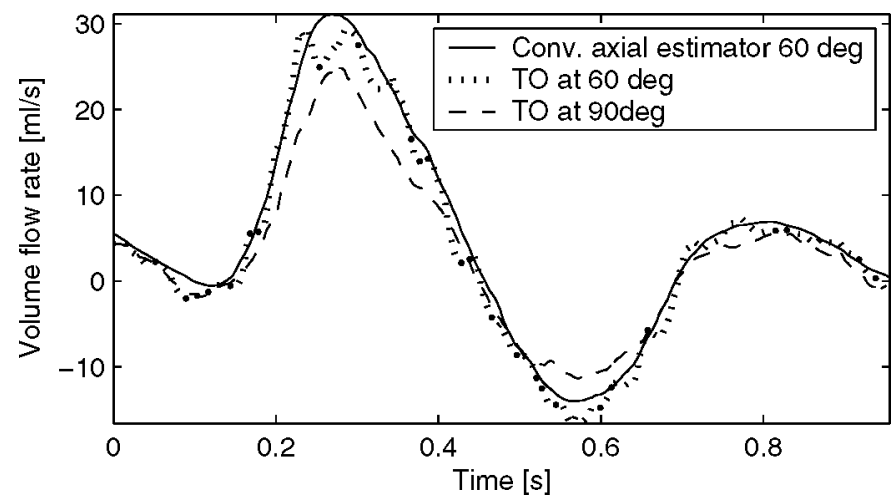

Fig. 20. The volume flow rate derived from the transverse velocity at $90^{\circ}$, the transverse velocity at $60^{\circ}$, and the axial velocity at $60^{\circ}$.

is derived from the transverse velocity profiles. The relative error between the two flow rate profiles at $60^{\circ}$ is found to be $13.4 \%$. The relative error between the flow rate derived from the transverse velocities at $90^{\circ}$ and the flow rate derived from the axial velocity at $60^{\circ}$ is found to be $27.8 \%$. Here the relative error $\alpha$ between the two volume profiles $Q_{r e f}(n)$ and $Q(n)$ of length $N$ is defined as:

$$
\alpha=\frac{\sum_{n=1}^{N}\left|Q_{\text {ref }}(n)-Q(n)\right|}{\sum_{n=1}^{N}\left|Q_{\text {ref }}(n)\right|},
$$

where the reference flow rate $Q_{r e f}(n)$ is the flow rate derived from the axial velocity estimate at $60^{\circ}$.

\section{Discussion}

A parameter study of the TO method has been performed, and the resulting relative standard deviation and relative bias have been calculated for a number of points in the parameter space. The parameter study shows that the TO method is sensitive to the angle between the ultrasound beam and the flow direction. At angles more than approximately $45^{\circ}$, the TO method can produce relatively good estimates of the transverse velocity. Thus, it is possible to estimate blood velocities at angles in which a conventional scanner fails to detect any velocity at all.

Furthermore, it has been shown that the TO method is capable of measuring the transverse velocity in an experimental setup for angles between $60^{\circ}$ and $90^{\circ}$ for both constant and pulsatile flow. Parabolic velocity profiles are easily detectable down to an angle of $60^{\circ}$ with a relative standard deviation and relative bias of approximately $10 \%$. For pulsatile flow, the characteristic profile of the flow in the femoral artery can be seen at both $90^{\circ}$ and $60^{\circ}$.

\section{CONCLUSIONS}

Future work should concentrate on the important issue of optimizing the echo-canceling filter. For small blood ves- 
sels, the clutter signal will dominate the blood signal even in the center of the vessel, and the mean subtraction filter will presumably not be sufficient to remove all the clutter signal.

Also, a clinical evaluation of the TO method has to be performed by an experienced sonographer. Different representative blood vessels have to be scanned, and a reliable reference velocity estimate can be obtained by tilting the ultrasound beam. The TO method can then be evaluated by comparing the reference one-dimensional velocity to the two-dimensional vector estimate.

\section{REFERENCES}

[1] M. D. Fox, "Multiple crossed-beam ultrasound Doppler velocimetry," IEEE Trans. Sonics Ultrason., vol. SU-25, pp. 281286, 1978.

[2] G. E. Trahey, J. W. Allison, and O. T. von Ramm, "Angle independent ultrasonic detection of blood flow," IEEE Trans. Biomed. Eng., vol. BME-34, pp. 965-967, 1987.

[3] V. L. Newhouse, D. Censor, T. Vontz, J. A. Cisneros, and B. B. Goldberg, "Ultrasound Doppler probing of flows transverse with respect to beam axis," IEEE Trans. Biomed. Eng., vol. BME-34, pp. $779-788,1987$.

[4] O. Bonnefous, "Measurement of the complete (3D) velocity vector of blood flows," in Proc. IEEE Ultrason. Symp., 1988, pp. 795-799.

[5] J. A. Jensen, "Directional velocity estimation using focusing along the flow direction: I: Theory and simulation," IEEE Trans. Ultrason., Ferroelect., Freq. Contr., vol. 50, pp. 857-872, July 2003.

6] P. Munk, "Estimation of the 2-D flow vector in ultrasonic imaging: A new approach," M.S. thesis, Department of Information Technology, Technical University of Denmark, Lyngby, Denmark, 1996.

[7] J. A. Jensen and P. Munk, "A new method for estimation of velocity vectors," IEEE Trans. Ultrason., Ferroelect., Freq. Contr., vol. 45, pp. 837-851, 1998.

[8] J. A. Jensen, "A new estimator for vector velocity estimation," IEEE Trans. Ultrason., Ferroelect., Freq. Contr., vol. 48, no. 4, pp. 886-894, 2001

[9] M. E. Anderson, "Spatial quadrature: A novel technique for multidimensional velocity estimation," in Proc. IEEE Ultrason. Symp., 1997, pp. 1233-1238.

[10] M. E. Anderson, "A heterodyning demodulation technique for spatial quadrature," in Proc. SPIE-Med. Imag., vol. 2, 2000, pp. $1487-1490$.

[11] J. Udesen and J. A. Jensen, "An in-vivo investigation of transverse flow estimation," Proc. SPIE-Progress Biomed. Opt. Imaging, vol. 5373, pp. 307-314, 2004.

[12] J. Udesen and J. A. Jensen, "Experimental investigation of transverse flow estimation using transverse oscillation," in Proc. IEEE Ultrason. Symp., 2003, pp. 1586-1589.

[13] J. A. Jensen and N. B. Svendsen, "Calculation of pressure fields from arbitrarily shaped, apodized, and excited ultrasound transducers," IEEE Trans. Ultrason., Ferroelect., Freq. Contr., vol. 39, pp. 262-267, 1992.

[14] J. A. Jensen, "Field: A program for simulating ultrasound systems," in Med. Biol. Eng. Comp., 10th Nordic-Baltic Conference on Biomedical Imaging, vol. 4, suppl. 1, pt. 1, pp. 351-353, 1996b.
[15] J. A. Jensen, O. Holm, L. J. Jensen, H. Bendsen, H. M. Pedersen, K. Salomonsen, J. Hansen, and S. Nikolov, "Experimental ultrasound system for real-time synthetic imaging," in Proc. IEEE Ultrason. Symp., 1999, pp. 1595-1599.

[16] J. W. Goodman, Introduction to Fourier Optics. 2nd ed. New York: McGraw-Hill, 1996.

[17] C. Kasai, K. Namekawa, A. Koyano, and R. Omoto, "Real-time two-dimensional blood flow imaging using an autocorrelation technique," IEEE Trans. Sonics Ultrason., vol. 32, pp. 458-463, 1985.

[18] J. A. Jensen, Estimation of Blood Velocities Using Ultrasound: A Signal Processing Approach. New York: Cambridge Univ. Press, 1996.

[19] D. H. Johnson and D. E. Dudgeon, Array Signal Processing. Concepts and Techniques. Englewood Cliffs, NJ: Prentice-Hall, 1993.

[20] K. V. Ramnarine, D. K. Nassiri, P. R. Hoskins, and J. Lubbers, "Validation of a new blood mimicking fluid for use in Doppler flow test objects," Ultrasound Med. Biol., vol. 24, pp. 451-459, 1998

[21] D. J. Acheson, Elementary Fluid Dynamics. Oxford: Clarendon, 1990

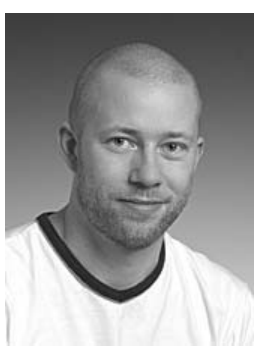

Jesper Udesen earned his Master of science degree in mathematics and physics from Roskilde University Center, Denmark, in March 2002. Since the summer of 2002 he has been employed as an industrial Ph.D. student at B-K Medical and at the Technical University of Denmark. His research interests include blood vector velocity estimation and beamforming techniques.

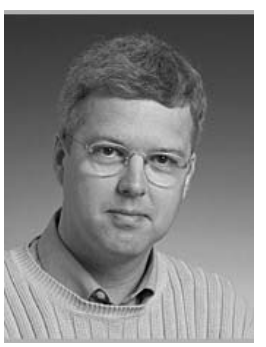

Jørgen Arendt Jensen (M'93-S'02) earned his Master of Science in electrical engineering in 1985 and the Ph.D. degree in 1989, both from the Technical University of Denmark. He received the Dr.Techn. degree from the university in 1996. He has published a number of papers on signal processing and medical ultrasound and the book "Estimation of Blood Velocities Using Ultrasound", Cambridge University Press in 1996. He is also the developer of the Field II simulation program. He has been a visiting scientist at Duke University, Stanford University, and the University of Illinois at UrbanaChampaign. He is currently full professor of Biomedical Signal Processing at the Technical University of Denmark at Ørsted•DTU and head of Center for Fast Ultrasound Imaging. He has given courses on blood velocity estimation at both Duke University and University of Illinois and teaches biomedical signal processing and medical imaging at the Technical University of Denmark. He has given several short courses on simulation, synthetic aperture imaging, and flow estimation at international scientific conferences. He has received several awards for his research. He is also the co-organizer of a new biomedical engineering education offered by the Technical University of Denmark and the University of Copenhagen. His research is centered around simulation of ultrasound imaging, synthetic aperture imaging and blood flow estimation and constructing systems for such imaging. 